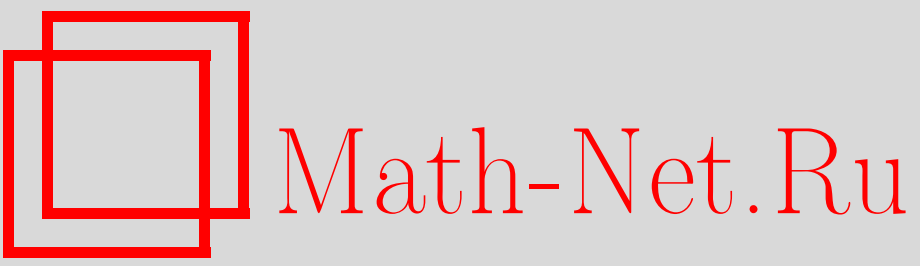

Н. А. Сердюкова, Зависимость сложности аппроксимации случайных полей от размерности, Теория вероятн. и ее примен., 2009, том 54, выпуск 2, 256-270

DOI: https://doi.org/10.4213/tvp2700

Использование Общероссийского математического портала Math-Net.Ru подразумевает, что вы прочитали и согласны с пользовательским соглашением

http://www . mathnet.ru/rus/agreement

Параметры загрузки:

IP : 3.85 .183 .62

26 апреля 2023 г., 18:20:08 


\section{ЗАВИСИМОСТЬ СЛОЖНОСТИ АППРОКСИМАЦИИ СЛУЧАЙНЫХ ПОЛЕЙ ОТ РАЗМЕРНОСТИ ${ }^{1)}$}

В настоящей статье рассматривается $\varepsilon$-аппроксимация $d$-параметрических случайных полей тензорного типа частичными суммами ряда, соответствующего разложению Карунена-Лоэва. При $d \rightarrow \infty$ изучено поведение информационной сложности $n(\varepsilon, d)$ аппроксимации с ошибкой, не превосходящей заданный уровень $\varepsilon$. Недавно М.А.Лифшицем и Е.В. Туляковой было доказано, что в рассматриваемой задаче наблюдается феномен «проклятия размерности». Целью данной статьи является получение точного асимптотического выражения для информационной сложности $n(\varepsilon, d)$.

Ключевые слова и фразы: случайные поля, гауссовские процессы, ошибка линейной аппроксимации, сложность, проклятие размерности.

1. Введение. Пусть $T$ - некоторое параметрическое множество. Предположим, что случайная функция $X(t), t \in T$, допускает представление в виде ряда

$$
X(t)=\sum_{k=1}^{\infty} \xi_{k} \varphi_{k}(t)
$$

где $\xi_{k}$ - случайные величины, $\varphi_{k}$ - детерминированные вещественные функции, а ряд сходится в среднем квадратическом при каждом $t \in T$. Более точная формулировка будет дана ниже. Для каждого конечного множества натуральных чисел $K \subset \mathbf{N}$ обозначим $X_{K}(t)=\sum_{k \in K} \xi_{k} \varphi_{k}(t)$. При решении многих задач бывает необходимо аппроксимировать $X$, например в $L_{2}$, процессом конечного ранга $X_{K}$, в связи с чем возникают естественные вопросы: какого размера должно быть множество $K$, чтобы обеспечить заданную точность аппроксимации? Среди всех множеств $K$ заданного размера, множество какого вида обеспечит наименьшую ошибку?

В настоящей статье мы ответим на первый из поставленных вопросов применительно к определенному классу случайных функций, а

\footnotetext{
${ }^{*}$ Weierstrass-Institut für Angewandte Analysis und Stochastik, Mohrenstr. 39, 10117 Berlin, Germany, e-mail: serdyuko@wias-berlin.de

1) Работа выполнена при поддержке РФФИ (грант № 05-01-00911) и РФФИ-ННИО (грант № 04-01-04000).
} 
именно, применительно к случайньм полям «тензорного» типа с параметрическими множествами высокой размерности. Полем типа «тензорного произведения» будем называть сепарабельное с нулевым средним случайное поле $X=\{X(t)\}_{t \in T}, T \subset \mathbf{R}^{d}$, с «прямоугольным» параметрическим множеством $T$ и ковариационной функцией $\mathscr{K}^{(d)}$, допускающими представление в виде произведения одинаковых сомножителей. А именно, $T=[0,1]^{d}$ и

$$
\mathscr{K}^{(d)}(s, t)=\prod_{l=1}^{d} \mathscr{K}_{l}\left(s_{l}, t_{l}\right)
$$

для любых $s=\left(s_{1}, \ldots, s_{d}\right), t=\left(t_{1}, \ldots, t_{d}\right)$, где $s_{l}, t_{l} \in[0,1]$. Очевидно, что интегральный оператор с ядром (1) является тензорным произведением интегральных операторов с ядрами $\mathscr{K}_{l}\left(s_{l}, t_{l}\right)$.

Пусть неотрицательная последовательность $\{\lambda(i)\}_{i \geqslant 1}$ Удовлетворяет условию

$$
\sum_{i=1}^{\infty} \lambda(i)^{2}<\infty
$$

и пусть функции $\left\{\varphi_{i}\right\}_{i>0}$ образуют полную ортонормированную систему в $L_{2}[0,1]$. Рассмотрим семейство случайных полей типа «тензорного произведения»

$$
\mathbf{X}=\left\{X^{(d)}(t), t \in[0,1]^{d}\right\}, \quad d=1,2, \ldots
$$

В силу многопараметрического разложения Карунена-Лоэва (см., например, [1]) семейство (3) может быть задано сходящимся п.н. в $L_{2}\left([0,1]^{d}\right)$ рядом

$$
\begin{aligned}
X^{(d)}(t) & =\sum_{k \in \mathbf{N}^{d}} \xi_{k} \prod_{l=1}^{d} \lambda\left(k_{l}\right) \prod_{l=1}^{d} \varphi_{k_{l}}\left(t_{l}\right) \\
& =\sum_{k_{1}=1}^{\infty} \cdots \sum_{k_{d}=1}^{\infty} \xi_{k_{1}, \ldots, k_{d}} \lambda\left(k_{1}\right) \cdots \lambda\left(k_{d}\right) \varphi_{k_{1}}\left(t_{1}\right) \cdots \varphi_{k_{d}}\left(t_{d}\right)
\end{aligned}
$$

$t=\left(t_{1}, \ldots, t_{d}\right) \in[0,1]^{d}$, где $\left\{\xi_{k}\right\}$ - массив некоррелированных случайных величин с нулевым средним и единичной дисперсией, а $\lambda\left(k_{l}\right)^{2}$ и $\varphi_{k_{l}}$ являются собственными значениями и собственными функциями семейства интегральных уравнений

$$
\lambda\left(k_{l}\right)^{2} \varphi_{k_{l}}\left(t_{l}\right)=\int_{0}^{1} \mathscr{K}_{l}\left(s_{l}, t_{l}\right) \varphi_{k_{l}}\left(s_{l}\right) \mathrm{d} s_{l}, \quad t_{l} \in[0,1], \quad l=1, \ldots, d,
$$

соответствующих «маргинальным» ковариационным операторам. Очевидно, что если выполнено условие $(2)$, то траектории $X^{(d)}$ принадлежат 
$L_{2}\left([0,1]^{d}\right)$ п.н. и система собственных чисел ковариационного оператора $X^{(d)}$ имеет вид

$$
\lambda_{k}^{2}=\prod_{l=1}^{d} \lambda\left(k_{l}\right)^{2}, \quad k \in \mathbf{N}^{d} .
$$

Как было отмечено в работе [27], разложение Карунена-Лоэва, или каноническое представление, случайной функции было предложено независимо Д. Д. Косамби [21], М. Лоэвом [23], К. Каруненом [19], [20], А. М. Обуховым [24] и В. С. Пугачевым [25].

В дальнейшем мы опускаем индекс $d$ и пишем $X(t)$ вместо $X^{(d)}(t)$. Для любого $n>0$ обозначим через $X_{n}$ частичные суммы ряда (4), отвечающие $n$ максимальным собственным числам. Нас интересует асимптотическое поведение ошибки аппроксимации в среднем процесса $X$ посредством $X_{n}$, а именно,

$$
e\left(X, X_{n} ; d\right):=\left(\mathbf{E}\left\|X-X_{n}\right\|_{L_{2}(T)}^{2}\right)^{1 / 2}, \quad d \rightarrow \infty .
$$

Далее мы будем рассматривать только $L_{2}(T)$-нормы, поэтому под символом $\|\cdot\|$ всегда будем подразумевать $\|\cdot\|_{L_{2}(T)}$. Хорошо известно (см., например, [6], [14] или [26]), что $X_{n}$ обеспечивает минимальную, среди всех линейных аппроксимаций порядка $n$, среднеквадратичную ошибку.

Поскольку мы собираемся исследовать семейство случайных функций, то более естественно изучать относительные ошибки, т.е. сравнивать величину ошибки с величиной самой функции.

Обозначим

$$
\Lambda:=\sum_{i=1}^{\infty} \lambda(i)^{2}
$$

тогда

$$
\mathbf{E}\|X\|^{2}=\sum_{k \in \mathbf{N}^{d}} \lambda_{k}^{2}=\Lambda^{d} .
$$

Определим относительную сложность среднеквадратичной аппроксимации следующим образом:

$$
n(\varepsilon, d):=\min \left\{n: \frac{e\left(X, X_{n} ; d\right)}{\left(\mathbf{E}\|X\|^{2}\right)^{1 / 2}} \leqslant \varepsilon\right\}=\min \left\{n: \mathbf{E}\left\|X-X_{n}\right\|^{2} \leqslant \varepsilon^{2} \Lambda^{d}\right\} .
$$

Заметим, что интересующее нас поведение сложности аппроксимации $n(\varepsilon, d)$ принадлежит к классу проблем, связанных с изучением зависимости сложности линейных многопараметрических задач от размерности (см. работы [30]-[33] и приведенные там ссылки).

Для изучения свойств (детерминированного!) массива собственных чисел (5) в [15] было предложено использовать вспомогательную вероятностную конструкцию. Мы также будем придерживаться этого подхода. 
Рассмотрим последовательность независимых одинаково распределенных случайных величин $\left\{U_{l}\right\}, l=1,2, \ldots$, общее распределение которых задано формулой

$$
\mathbf{P}\left(U_{l}=-\ln \lambda(i)\right)=\frac{\lambda(i)^{2}}{\Lambda}, \quad i=1,2, \ldots
$$

Если выполнено условие

$$
\sum_{i=1}^{\infty}|\ln \lambda(i)|^{3} \lambda(i)^{2}<\infty
$$

то, очевидно, $\mathbf{E}\left|U_{l}\right|^{3}<\infty$.

Обозначим через $M$ и $\sigma^{2}$ математическое ожидание и дисперсию $U_{l}$ соответственно. Ясно, что

$$
M=-\sum_{i=1}^{\infty} \ln \lambda(i) \frac{\lambda(i)^{2}}{\Lambda}, \quad \sigma^{2}=\sum_{i=1}^{\infty}|\ln \lambda(i)|^{2} \frac{\lambda(i)^{2}}{\Lambda}-M^{2} .
$$

Тогда третий центральный момент $U_{l}$ задается соотношением

$$
\alpha^{3}:=\mathbf{E}\left(U_{l}-M\right)^{3}=-\sum_{i=1}^{\infty}(\ln \lambda(i))^{3} \frac{\lambda(i)^{2}}{\Lambda}-3 M \sigma^{2}-M^{3} .
$$

Если выполнено (7), то $|M|<\infty, 0 \leqslant \sigma^{2}<\infty$ и $|\alpha|<\infty$.

В дальнейшем «взрывающийся» коэффициент

$$
\mathscr{E}:=\Lambda e^{2 M}
$$

будет играть значительную роль. В [15] показано, что, в силу вогнутости логарифмической функции, $\mathscr{E}>1$, за исключением вырожденного случая, когда число положительных собственных чисел равно нулю или единице. Другими словами, $\mathscr{E}=1$ тогда и только тогда, когда $\sigma=0$. Мы исключим этот случай из дальнейшего рассмотрения.

В [15] получен следующий результат (теорема 3.2 ).

Теорема 1.1. Предположим, ито последовательность $\{\lambda(i)\}, i=$ $1,2, \ldots$, удовлетворяет условию

$$
\sum_{i=1}^{\infty}|\ln \lambda(i)|^{2} \lambda(i)^{2}<\infty
$$

Тогда для каждого $\varepsilon \in(0,1)$ выполнено

$$
\lim _{d \rightarrow \infty} \frac{\ln n(\varepsilon, d)-d \ln \mathscr{E}}{\sqrt{d}}=2 q,
$$

где квантиль $q=q(\varepsilon)$ есть решение уравнения

$$
1-\Phi\left(\frac{q}{\sigma}\right)=\varepsilon^{2} .
$$


Авторы [15] предположили, что при более сильных условиях на последовательность $\{\lambda(i)\}$ можно доказать, что

$$
n(\varepsilon, d) \approx \frac{C(\varepsilon) \mathscr{E}^{d} e^{2 q \sqrt{d}}}{\sqrt{d}}, \quad d \rightarrow \infty .
$$

Мы покажем, что выполнено даже несколько более точное утверждение.

2. Основной результат. Нам придется отдельно рассматривать два случая в зависимости от природы распределения величин $U_{l}$. Доказательство и конечный результат будут зависеть от того, является это распределение решетчатым или нет.

Напомним, что дискретное распределение случайной величины $U$ называется решетчатым, если существуют такие числа $a$ и $h>0$, что все возможные значения $U$ могут быть представлены в виде $a+\nu h$, где $\nu$ принимает целые значения. Число $h$ называется шагом распределения. В дальнейшем при рассмотрении решетчатого случая мы предполагаем, что $h$ является максимальным шагом распределения, а именно что невозможно представить все значения $U_{l}$ в виде $b+\nu h_{1}$ с некоторыми $b$ и $h_{1}>h$.

Из (6) следует, что величины $U_{l}$ имеют решетчатое распределение тогда и только тогда, когда $\lambda(i)=C e^{-n_{i} h}$ для некоторых положительных $C, h$ и $n_{i} \in \mathbf{N}$. Мы будем называть эту ситуацию решетчатым случаем и будем предполагать, что $h$ выбрано максимально возможным. В противном случае мы будем говорить, что имеет место нерешетчатьий случай.

Теорема 2.1. Пусть последовательность $\{\lambda(i)\}, i=1,2, \ldots$, удовлетворяет условию (7).

Тогда для каждого $\varepsilon \in(0,1)$ выполнено

$$
n(\varepsilon, d)=K \phi\left(\frac{q}{\sigma}\right) \mathscr{E} d e^{2 q \sqrt{d}} d^{-1 / 2}(1+o(1)), \quad d \rightarrow \infty,
$$

əде

$\phi(x)=\frac{1}{\sqrt{2 \pi}} e^{-x^{2} / 2}, \quad K= \begin{cases}h\left(\sigma\left(1-e^{-2 h}\right)\right)^{-1} & \text { в решетчатом случае, } \\ (2 \sigma)^{-1} & \text { в нерешетчатом случае },\end{cases}$ иквантиль $q=q(\varepsilon)$ определена в (9).

3 а м е ч а н и е. 1) Мы видим, что сложность аппроксимации возрастает экспоненциально, когда $d \rightarrow \infty$. Это явление обычно называют «проклятием размерности» (curse of dimensionality) или «intractability». См., например, [26] и [31]. Понятие «проклятия размерности» восходит, по крайней мере, к работам Р. Беллмана [4]. 
2) По правилу Лопиталя

$$
\lim _{h \rightarrow 0} \frac{h}{1-e^{-2 h}}=\frac{1}{2},
$$

так что формулы для $K$ согласованы между собой при $h \rightarrow 0$.

Д о к а з а т е л ь с т в о. Определим $\zeta=\zeta(\varepsilon, d)$ как максимальное положительное число такое, что сумма собственных чисел, меньших чем $\zeta^{2}$, удовлетворяет неравенству

$$
\sum_{k \in \mathbf{N}^{d}: \lambda_{k}<\zeta} \lambda_{k}^{2} \leqslant \varepsilon^{2} \Lambda^{d}
$$

Определим решетчатое множество в $\mathbf{N}^{d}$ следующим образом:

$$
\mathrm{A}=\mathrm{A}(\varepsilon, d):=\left\{k \in \mathbf{N}^{d}: \lambda_{k} \geqslant \zeta\right\}=\left\{k \in \mathbf{N}^{d}: \prod_{l=1}^{d} \lambda\left(k_{l}\right) \geqslant \zeta\right\}
$$

Поскольку $\lambda_{k}>0$ для любого $k \in \mathrm{A}$, то

$$
\begin{aligned}
& n(\varepsilon, d)=\operatorname{card}(\mathrm{A})=\sum_{k \in \mathrm{A}} \frac{\lambda_{k}^{2}}{\lambda_{k}^{2}} \\
& =\sum_{k \in \mathbf{N}^{d}:-\sum \ln \lambda\left(k_{l}\right) \leqslant-\ln \zeta} \Lambda^{d} \exp \left\{-2 \sum_{l=1}^{d} \ln \lambda\left(k_{l}\right)\right\} \prod_{l=1}^{d} \mathbf{P}\left(U_{l}=-\ln \lambda\left(k_{l}\right)\right) \\
& =\Lambda^{d} \mathbf{E} \exp \left\{2 \sum_{l=1}^{d} U_{l}\right\} \mathbf{I}\left\{\sum_{l=1}^{d} U_{l} \leqslant-\ln \zeta\right\} .
\end{aligned}
$$

Для центрированных и нормированных сумм $Z_{d}=\left(\sum_{l=1}^{d} U_{l}-d M\right) /(\sigma \sqrt{d})$ выполнено

$$
\left\{\sum_{l=1}^{d} U_{l} \leqslant-\ln \zeta\right\}=\left\{Z_{d} \leqslant \theta\right\},
$$

где

$$
\theta=\theta(\varepsilon, d)=-\frac{\ln \zeta+d M}{\sigma \sqrt{d}} .
$$

Покажем теперь, что $\theta$ допускает полезную вероятностную интерпретацию в терминах случайных величин $U_{l}$ и их сумм. Действительно, применяя лемму 3.1 из [15], мы имеем для любых $d \in \mathbf{N}$ и $z \in \mathbf{R}^{1}$ :

$$
\begin{aligned}
\sum_{k \in \mathbf{N}^{d}: \lambda_{k}<z} \lambda_{k}^{2} & =\Lambda^{d} \mathbf{P}\left(\sum_{l=1}^{d} U_{l}>-\ln z\right) \\
& =\Lambda^{d} \mathbf{P}\left(Z_{d}>-\frac{\ln z+d M}{\sigma \sqrt{d}}\right)=\Lambda^{d} \mathbf{P}\left(Z_{d}>\theta_{z}\right),
\end{aligned}
$$


где

$$
\theta_{z}=-\frac{\ln z+d M}{\sigma \sqrt{d}} .
$$

Зафиксируем $\varepsilon \in(0,1)$. Заметим, что $\sum_{k \in \mathbf{N}^{d}: \lambda_{k}<z} \lambda_{k}^{2} \leqslant \varepsilon^{2} \Lambda^{d}$ тогда и только тогда, когда $\mathbf{P}\left(Z_{d}>\theta_{z}\right) \leqslant \varepsilon^{2}$. Поэтому величина $\theta=\theta(\varepsilon, d)$, определенная равенством (10), является $\left(1-\varepsilon^{2}\right)$-квантилью распределения $Z_{d}$, а именно,

$$
\theta(\varepsilon, d)=\min \left\{\theta: \mathbf{P}\left(Z_{d}>\theta\right) \leqslant \varepsilon^{2}\right\}=\min \left\{\theta: \mathbf{P}\left(Z_{d} \leqslant \theta\right)>1-\varepsilon^{2}\right\} .
$$

Обозначим через $q=q(\varepsilon)$ квантиль функции распределения нормального закона, выбранную из уравнения (9). Тогда, в силу центральной предельной теоремы,

$$
\theta(d, \varepsilon) \rightarrow \frac{q(\varepsilon)}{\sigma}, \quad d \rightarrow \infty
$$

для каждого фиксированного $\varepsilon \in(0,1)$.

Теперь мы можем вернуться к изучению сложности аппроксимации. Мы получили, что

$$
\begin{aligned}
n(\varepsilon, d) & =\mathscr{E}^{d} \mathbf{E} \exp \left\{2 \sigma \sqrt{d} Z_{d}\right\} \mathbf{I}\left\{Z_{d} \leqslant \theta\right\} \\
& =\mathscr{E}^{d} \exp \{2 \sigma \sqrt{d} \theta\} \int_{-\infty}^{\theta} \exp \{2 \sigma \sqrt{d}(z-\theta)\} \mathrm{d} F_{d}(z),
\end{aligned}
$$

где $F_{d}(z)=\mathbf{P}\left(Z_{d}<z\right)$ и $\mathscr{E}$ определено в $(8)$.

Обозначим

$$
\Psi_{d}(z):=\exp \{2 \sigma \sqrt{d}(z-\theta)\} .
$$

Интегрируя по частям, получаем

$$
\int_{-\infty}^{\theta} \Psi_{d}(z) \mathrm{d}\left[F_{d}(z)-F_{d}(\theta)\right]=\int_{-\infty}^{\theta}\left[-F_{d}(z)+F_{d}(\theta)\right] \mathrm{d} \Psi_{d}(z) .
$$

Н е р е ш е т ч а ты й с л у ч а й. В этой части доказательства мы будем предполагать, что распределение случайных величин $U_{l}$ не является решетчатым. Это имеет место в наиболее интересных случаях, таких как броуновский лист (поле Винера-Ченцова), броуновская подушка, поле Хёффдинга-Блума-Кифера-Розенблатта (см. п. 3).

В силу того, что выполнено (7), мы можем применить теорему Крамера-Эссеена (см. [10, § 42, теорема 2], [17, гл. V, § 7, теорема 21] либо [16, гл. VI, $\S 3$, теорема 4]), откуда немедленно получаем

$$
\begin{aligned}
& \int_{-\infty}^{\theta}\left[-F_{d}(z)+F_{d}(\theta)\right] \mathrm{d} \Psi_{d}(z)=\int_{-\infty}^{\theta}[-\Phi(z)+\Phi(\theta)] \mathrm{d} \Psi_{d}(z) \\
& \quad+\frac{\alpha^{3}}{6 \sigma^{3} \sqrt{2 \pi d}} \int_{-\infty}^{\theta}\left[\left(z^{2}-1\right) e^{-z^{2} / 2}-\left(\theta^{2}-1\right) e^{-\theta^{2} / 2}\right] \mathrm{d} \Psi_{d}(z)+o\left(\frac{1}{\sqrt{d}}\right) \\
& \quad=I_{1}+I_{2}-I_{3}-I_{4}+o\left(\frac{1}{\sqrt{d}}\right)
\end{aligned}
$$


где $\Phi(\cdot)$ — стандартная нормальная функция распределения и

$$
\begin{aligned}
I_{1} & :=\int_{-\infty}^{\theta}[-\Phi(z)+\Phi(\theta)] \mathrm{d} \Psi_{d}(z), \\
I_{2} & :=\frac{\alpha^{3}}{6 \sigma^{3} \sqrt{2 \pi d}} \int_{-\infty}^{\theta} z^{2} e^{-z^{2} / 2} \mathrm{~d} \Psi_{d}(z), \\
I_{3} & :=\frac{\alpha^{3}}{6 \sigma^{3} \sqrt{2 \pi d}} \int_{-\infty}^{\theta} e^{-z^{2} / 2} \mathrm{~d} \Psi_{d}(z), \\
I_{4} & :=\frac{\alpha^{3}}{6 \sigma^{3} \sqrt{2 \pi d}}\left(\theta^{2}-1\right) e^{-\theta^{2} / 2} \sim \frac{\alpha^{3}}{6 \sigma^{3} \sqrt{2 \pi d}}\left(\left(\frac{q}{\sigma}\right)^{2}-1\right) e^{-q^{2} / 2 \sigma^{2}}, \quad d \rightarrow \infty
\end{aligned}
$$

(последнее соотношение следует из (11)). Поскольку $\mathrm{d} \Psi_{d}(z)=$ $2 \sigma \sqrt{d} \Psi_{d}(z) \mathrm{d} z$, то после замены переменных $I_{2}$ примет вид

$I_{2}=I_{2}(d, \theta)=\frac{\alpha^{3}}{3 \sigma^{2} \sqrt{2 \pi d}} \int_{0}^{\infty}\left(\theta-\frac{y}{\sqrt{d}}\right)^{2} \exp \left\{-\frac{1}{2}\left(\theta-\frac{y}{\sqrt{d}}\right)^{2}\right\} \exp \{-2 \sigma y\} \mathrm{d} y$,

где $y=-\sqrt{d}(z-\theta)$.

Для любого $d=1,2, \ldots$ верно неравенство

$$
0 \leqslant\left(\theta-\frac{y}{\sqrt{d}}\right)^{2} \exp \left\{-\frac{1}{2}\left(\theta-\frac{y}{\sqrt{d}}\right)^{2}\right\} \leqslant(|\theta|+y)^{2} .
$$

Эта оценка дает нам суммируемую мажоранту, необходимую для применения теоремы Лебега. Учитывая (11) и переходя к пределу под знаком интеграла, мы получаем, что при $d \rightarrow \infty$

$$
I_{2}(d, \theta)=\frac{\alpha^{3}}{6 \sigma^{3} \sqrt{2 \pi d}}\left(\frac{q}{\sigma}\right)^{2} e^{-q^{2} /\left(2 \sigma^{2}\right)}(1+o(1)) .
$$

Аналогично,

$$
I_{3}(d, \theta)=\frac{\alpha^{3}}{6 \sigma^{3} \sqrt{2 \pi d}} e^{-q^{2} /\left(2 \sigma^{2}\right)}(1+o(1)) .
$$

Таким образом, мы получили, что $\sqrt{d} I_{4}=\sqrt{d}\left(I_{2}-I_{3}\right)(1+o(1))$, следовательно, $I_{2}-I_{3}-I_{4}=o(1 / \sqrt{d})$.

Рассмотрим основной интеграл $I_{1}$ :

$$
\begin{aligned}
I_{1} & =I_{1}(d, \theta)=\int_{-\infty}^{\theta}[-\Phi(z)+\Phi(\theta)] \mathrm{d} \Psi_{d}(z) \\
& =\frac{1}{\sqrt{2 \pi}} \int_{-\infty}^{\theta} \exp \{2 \sigma \sqrt{d}(z-\theta)\} \exp \left\{-\frac{z^{2}}{2}\right\} \mathrm{d} z \\
& =\frac{1}{\sqrt{2 \pi d}} \int_{0}^{\infty} \exp \left\{-\frac{1}{2}\left(\theta-\frac{y}{\sqrt{d}}\right)^{2}\right\} \exp \{-2 \sigma y\} \mathrm{d} y \\
& \sim \frac{1}{2 \sigma \sqrt{2 \pi d}} e^{-q^{2} /\left(2 \sigma^{2}\right)}, \quad d \rightarrow \infty .
\end{aligned}
$$


Таким образом, мы получили желаемую оценку

$$
n(\varepsilon, d)=\frac{\mathscr{E}^{d} \exp \{2 q \sqrt{d}\}}{2 \sigma \sqrt{d}} \frac{1}{\sqrt{2 \pi}} \exp \left\{-\frac{q^{2}}{2 \sigma^{2}}\right\}(1+o(1)) .
$$

Р е ш е т ч а т ы й с л у ч а й. Теперь мы будем действовать в предположении, что последовательность $\left\{U_{l}\right\}$ имеет решетчатое распределение.

Пусть случайная величина $U_{l}$ принимает значения вида $\widetilde{a}+\nu h$, $\nu=0, \pm 1, \pm 2, \ldots$, где $\widetilde{a}=M+a-$ сдвиг, а $h-$ максимальный шаг распределения. Тогда все возможные значения суммы $Z_{d}$ представимы в виде

$$
\frac{d a+\nu h}{\sigma \sqrt{d}}, \quad \nu=0, \pm 1, \pm 2, \ldots
$$

Введем в рассмотрение функцию

$$
S(x)=[x]-x+\frac{1}{2}
$$

где $[x]$ обозначает, как обычно, целую часть $x$, и определим

$$
S_{d}(x)=\frac{h}{\sigma} S\left(\frac{x \sigma \sqrt{d}-d a}{h}\right)
$$

Пусть $F_{d}(z)$ определена как прежде. Тогда если выполнено $(7)$, то результат Эссеена (см. [10, 443 , теорема 1]) влечет равенство

$$
F_{d}(z)-\Phi(z)=\frac{e^{-z^{2} / 2}}{\sqrt{2 \pi}}\left(\frac{S_{d}(z)}{\sqrt{d}}-\frac{\alpha^{3}\left(z^{2}-1\right)}{6 \sigma^{3} \sqrt{d}}\right)+o\left(\frac{1}{\sqrt{d}}\right)
$$

равномерно по $z$.

Сравнивая с (12), мы видим, что нам нужно оценить только дополнительное слагаемое

$$
\begin{aligned}
J & :=\frac{1}{\sqrt{2 \pi d}} \int_{-\infty}^{\theta}\left[-S_{d}(z) e^{-z^{2} / 2}+S_{d}(\theta) e^{-\theta^{2} / 2}\right] \mathrm{d} \Psi_{d}(z) \\
& =\frac{1}{\sqrt{2 \pi d}} \int_{-\infty}^{\theta} \Psi_{d}(z) \mathrm{d}\left(S_{d}(z) e^{-z^{2} / 2}\right)=J_{1}-J_{2}+J_{3},
\end{aligned}
$$

где

$$
\begin{aligned}
& J_{1}:=\frac{1}{\sqrt{2 \pi d}} \int_{-\infty}^{\theta} \Psi_{d}(z) S_{d}^{\prime}(z) e^{-z^{2} / 2} \mathrm{~d} z \\
& J_{2}:=\frac{1}{\sqrt{2 \pi d}} \int_{-\infty}^{\theta} \Psi_{d}(z) S_{d}(z) z e^{-z^{2} / 2} \mathrm{~d} z
\end{aligned}
$$


и $J_{3}$ - «дискретная часть», которая определена следующим образом. Заметим, что $S(x)$ является периодической функцией с единичным периодом, поэтому $S_{d}(x)$ обладает периодом $h /(\sigma \sqrt{d})$ и имеет скачки в точках вида $\{(k h+d a) /(\sigma \sqrt{d}), k \in \mathbf{Z}\}$. Если $\theta$ принадлежит этой решетке, то существует целое $k^{\prime}$ такое, что $\theta=\left(k^{\prime} h+d a\right) /(\sigma \sqrt{d})$. Следовательно, можно интегрировать разрывную часть интеграла $J$ по отношению к дираковской мере $(h / \sigma) \delta_{(k h+d a) /(\sigma \sqrt{d})}$. Тогда

$$
J_{3}:=\frac{1}{\sqrt{2 \pi d}} \frac{h}{\sigma} \sum_{k=-\infty}^{k^{\prime}} \Psi_{d}\left(\frac{k h+d a}{\sigma \sqrt{d}}\right) \exp \left\{-\frac{1}{2}\left(\frac{k h+d a}{\sigma \sqrt{d}}\right)^{2}\right\} .
$$

Оценим сначала интеграл $J_{1}$. В тех точках, где производная $S_{d}^{\prime}(z)$ имеет смысл, можно легко вычислить, что

$$
S_{d}^{\prime}(z)=\frac{h}{\sigma} S\left(\frac{z \sigma \sqrt{d}-d a}{h}\right)=-\sqrt{d}
$$

Тогда, подобно нерешетчатому случаю, по теореме Лебега мы имеем

$$
\begin{aligned}
J_{1} & =\frac{-\sqrt{d}}{\sqrt{2 \pi d}} \int_{-\infty}^{\theta} \exp \{2 \sigma \sqrt{d}(z-\theta)\} \exp \left\{\frac{-z^{2}}{2}\right\} \mathrm{d} z \\
& =\frac{-1}{\sqrt{2 \pi d}} \int_{0}^{\infty} \exp \left\{-\frac{1}{2}\left(\theta-\frac{y}{\sqrt{d}}\right)^{2}\right\} \exp \{-2 \sigma y\} \mathrm{d} y \\
& \sim \frac{-1}{2 \sigma \sqrt{2 \pi d}} e^{-q^{2} /\left(2 \sigma^{2}\right)}, \quad d \rightarrow \infty,
\end{aligned}
$$

следовательно, $\sqrt{d} J_{1}=-\sqrt{d} I_{1}(1+o(1))$.

Что касается интеграла $J_{2}$, то он при достаточно больших $d$ становится пренебрежимо мал. Действительно,

$$
\begin{aligned}
J_{2}= & \frac{1}{\sqrt{2 \pi d}} \int_{-\infty}^{\theta} \exp \{2 \sigma \sqrt{d}(z-\theta)\} S_{d}(z) z \exp \left\{\frac{-z^{2}}{2}\right\} \mathrm{d} z \\
= & \frac{1}{\sqrt{2 \pi d}} \frac{1}{\sqrt{d}} \int_{0}^{\infty} \exp \left\{-\frac{1}{2}\left(\theta-\frac{y}{\sqrt{d}}\right)^{2}\right\}\left(\theta-\frac{y}{\sqrt{d}}\right) \\
& \times S_{d}\left(\theta-\frac{y}{\sqrt{d}}\right) \exp \{-2 \sigma y\} \mathrm{d} y \\
\leqslant & \frac{3 h}{2 \sigma d \sqrt{2 \pi}} \int_{0}^{\infty} \exp \left\{-\frac{1}{2}\left(\theta-\frac{y}{\sqrt{d}}\right)^{2}\right\}\left(\theta-\frac{y}{\sqrt{d}}\right) \exp \{-2 \sigma y\} \mathrm{d} y \\
\sim & \frac{3 h}{4 \sigma^{2} d \sqrt{2 \pi}}\left(\frac{q}{\sigma}\right)^{2} e^{-q^{2} /\left(2 \sigma^{2}\right)}, \quad d \rightarrow \infty .
\end{aligned}
$$

И, разумеется, $J_{2}=o(1 / \sqrt{d})$. 
Теперь мы перейдем к рассмотрению наиболее важного слагаемого $J_{3}$ :

$$
\begin{aligned}
J_{3} & =\frac{1}{\sqrt{2 \pi d}} \frac{h}{\sigma} \sum_{k=-\infty}^{k^{\prime}} \exp \left\{2 \sigma \sqrt{d}\left(\frac{k h+d a}{\sigma \sqrt{d}}-\theta\right)\right\} \exp \left\{-\frac{1}{2}\left(\frac{k h+d a}{\sigma \sqrt{d}}\right)^{2}\right\} \\
& =\frac{1}{\sqrt{2 \pi d}} \frac{h}{\sigma} \sum_{k=-\infty}^{k^{\prime}} \exp \left\{2 h\left(k-k^{\prime}\right)\right\} \exp \left\{-\frac{1}{2}\left(\frac{k h+d a}{\sigma \sqrt{d}}\right)^{2}\right\} \\
& =\frac{1}{\sqrt{2 \pi d}} \frac{h}{\sigma} \sum_{l=0}^{\infty} \exp \{-2 h l\} \exp \left\{-\frac{1}{2}\left(\frac{\left(k^{\prime}-l\right) h+d a}{\sigma \sqrt{d}}\right)^{2}\right\} \\
& =\frac{1}{\sqrt{2 \pi d}} \frac{h}{\sigma} \sum_{l=0}^{\infty} \exp \{-2 h l\} \exp \left\{-\frac{1}{2}\left(\theta-\frac{l h}{\sigma \sqrt{d}}\right)^{2}\right\} \\
& \sim \frac{1}{\sigma \sqrt{d}} \frac{h}{\left(1-e^{-2 h}\right)} \frac{1}{\sqrt{2 \pi}} e^{-q^{2} /\left(2 \sigma^{2}\right)}, \quad d \rightarrow \infty .
\end{aligned}
$$

Таким образом,

$$
\sqrt{d} J_{3}=\sqrt{d} \frac{2 h}{\left(1-e^{-2 h}\right)} I_{1}(1+o(1)) .
$$

Объединяя оценки (13), (14) и (15), мы получаем

$$
n(\varepsilon, d)=\frac{\mathscr{E}^{d} e^{2 q \sqrt{d}}}{\sigma \sqrt{d}} \frac{h}{\left(1-e^{-2 h}\right)} \frac{1}{\sqrt{2 \pi}} e^{-q^{2} /\left(2 \sigma^{2}\right)}(1+o(1)), \quad d \rightarrow \infty .
$$

Теорема 2.1 доказана.

3. Приложение. Примеры случайных полей типа «тензорного произведения». Приведем примеры полей, к которым может быть применен полученный общий результат.

Поле Винера-Ченцова. Полем Винера-Ченцова $W^{(d)}$ называется (см. [22]) гауссовская случайная функция с нулевым средним и ковариационной функцией, равной произведению ковариационных функций, соответствующих стандартному броуновскому движению $W$ :

$$
\mathscr{K}_{W}^{(d)}(s, t)=\prod_{l=1}^{d} \min \left\{s_{l}, t_{l}\right\}, \quad s=\left(s_{1}, \ldots, s_{d}\right), t=\left(t_{1}, \ldots, t_{d}\right) \in T
$$

Поэтому «маргинальные» собственные числа имеют вид

$$
\lambda_{W}(i)^{2}=\frac{1}{(\pi(i-1 / 2))^{2}}, \quad i=1,2, \ldots
$$


Броуновская подушка. Броуновской подушкой $B^{(2)}$ называется гауссовская случайная функция с нулевым средним и ковариационной функцией, равной произведению ковариационных функций двух независимых стандартных броуновских мостов $B(t)=W(t)-t W(1)$, а именно,

$$
\mathscr{K}_{B}^{(2)}(s, t)=\prod_{l=1}^{2}\left(\min \left\{s_{l}, t_{l}\right\}-s_{l} t_{l}\right), \quad s, t \in[0,1]^{2} .
$$

Соответственно, «маргинальные» собственные числа (см. [2]) равны

$$
\lambda_{B}(i)^{2}=(\pi i)^{-2}, \quad i=1,2, \ldots
$$

В англоязычной литературе чаще чем «Brownian pillow» или «Wiener pillow» используются термины «completely tucked Brownian sheet» (см. [28]) или «tied-down Kiefer process» (см. [7]). Дж. Блум, Дж. Кифер и М.Розенблатт в работе [5] ввели броуновскую подушку (а также ее обобщение на случай $d>2$ ) как предельное распределение некоторого функционала от эмпирического процесса, используемого при непараметрическом тестировании независимости, - так называемого «independence empirical process» (см. [28]). Поэтому $d$-параметрическое обобщение броуновской подушки часто называют процессом Хёффдинга-Блума-Кифера-Розенблатта (см., например, [13]). Упоминание имени Хёффдинга в названии процесса связано с тем, что в [5] изучался тест, эквивалентный ранее предложенному В. Хёффдингом в [12]. Однако предельное распределение, вид ковариационной функции, собственные числа и собственные функции соответствующего интегрального уравнения были получены именно в [5]. Многомерные обобщения броуновской подушки изучались в $[9]$ и в [8].

Центрированные гауссовские процессы. В некоторых статистических задачах бывает удобно использовать центрированные эмпирические процессы и соответствующие им предельные гауссовские процессы.

Для произвольного гауссовского процесса $X=\{X(t), t \in[0,1]\}$ определим центрированный процесс $X^{c}$ как

$$
X^{c}(t):=X(t)-\int_{0}^{1} X(u) \mathrm{d} u .
$$

Центрированный броуновский мост $B^{c}$, известный в литературе также как процесс Ватсона, был введен в [29] для непараметрической проверки гипотезы согласия на круге. Ватсон показал, что ковариационная функция центрированного броуновского моста задается формулой

$$
\mathscr{K}_{B^{c}}(s, t)=\min \{s, t\}-s t+\frac{1}{2}\left(s^{2}+t^{2}-s-t\right)+\frac{1}{12}, \quad s, t \in[0,1],
$$


а ковариационный оператор с таким ядром обладает «двойным спектром», т.е.

$$
\lambda_{B^{c}}(2 i)^{2}=\lambda_{B^{c}}(2 i-1)^{2}=(2 \pi i)^{-2}, \quad i=1,2, \ldots
$$

Ковариационная функция центрированного броуновского движения $W^{c}$ имеет вид

$$
\mathscr{K}_{W^{c}}(s, t)=\min \{s, t\}+\frac{1}{2}\left(s^{2}+t^{2}\right)-s-t+\frac{1}{3}, \quad s, t \in[0,1],
$$

а его собственные числа совпадают с собственными числами стандартного броуновского моста, т.е.

$$
\lambda_{W^{c}}(i)^{2}=\lambda_{B}(i)^{2}=(\pi i)^{-2}, \quad i=1,2, \ldots,
$$

что согласуется с известным равенством по распределению $L_{2}$-норм броуновского моста и центрированного броуновского движения (см. [3]).

Центрированный интегрированный броуновский мост

$$
\breve{B}(t)=\bar{B}(t)-\int_{0}^{1} \bar{B}(u) \mathrm{d} u
$$

где $\bar{B}(t)=\int_{0}^{t} B(u) \mathrm{d} u, t \in[0,1]$, изучался в контексте проверки гипотезы согласия и асимптотики малых уклонений относительно $L_{2}$-нормы в [1] и [3], где были найдены его ковариационная функция

$\mathscr{K}_{\breve{B}}(s, t)=\frac{s t \min \{s, t\}}{2}-\frac{\min \{s, t\}^{3}}{6}-\frac{(s t)^{2}}{4}-\frac{s^{2}+t^{2}}{6}-\frac{s^{4}+t^{4}}{24}+\frac{s^{3}+t^{3}}{6}+\frac{1}{45}$, $s, t \in[0,1]$, и собственные числа $\lambda_{\breve{B}}(i)^{2}=(\pi i)^{-4}, i=1,2, \ldots$.

Многопараметрические обобщения процесса АндерсонаДарлинга. «Тензорным произведением» процессов Андерсона-Дарлинга $A^{(d)}(t), t \in[0,1]^{d}$, называется гауссовский процесс с нулевым средним и ковариационной функцией

$$
\mathscr{K}_{A}^{(d)}(s, t)=\prod_{l=1}^{d} \frac{\min \left\{s_{l}, t_{l}\right\}-s_{l} t_{l}}{\sqrt{s_{l}\left(1-s_{l}\right)} \sqrt{t_{l}\left(1-t_{l}\right)}}, \quad s_{l}, t_{l} \in[0,1] .
$$

Собственные числа его ковариационного оператора имеют вид

$$
\lambda_{k}^{2}=\prod_{l=1}^{d} \frac{1}{k_{l}\left(k_{l}+1\right)}, \quad k=\left(k_{1}, \ldots, k_{d}\right) \in \mathbf{N}^{d}
$$

В одномерном случае процесс Андерсона-Дарлинга совпадает по распределению с $B(t) / \sqrt{t(1-t)}, t \in[0,1]$, и был определен в [2] в контексте проверки гипотезы согласия. Т. Андерсон и Д. Дарлинг нашли его ковариационную функцию и точный спектр. 
В [18] введено другое многопараметрическое обобщение процесса Андерсона-Дарлинга, определенное как гауссовский процесс с нулевым средним и ковариационной функцией

$$
\mathscr{K}_{A}^{\mu}(s, t)=\left(\frac{\min \{s, t\}-s t}{\sqrt{s(1-s)} \sqrt{t(1-t)}}\right)^{\mu}, \quad s, t \in[0,1], \quad \mu>0 .
$$

Собственные числа его ковариационного оператора задаются соотношением

$$
\lambda_{\mu, j}^{2}=\frac{\mu}{(\mu+j-1)(\mu+j)}, \quad j=1,2, \ldots .
$$

При целых положительных значениях параметра $\mu$ так определенное поле (точнее, квадрат его $L_{2}$-нормы) оказывается предельным распределением для статистик типа Крамера-фон Мизеса.

Настоящая статья была частично написана во время пребывания автора в Институте математической стохастики Университета Георга Августа, Гёттинген. Хочется особенно поблагодарить профессора М. А. Лифшица за постановку данной задачи и постоянную поддержку, а также профессора М. Денкера за содействие и обеспечение прекрасных условий для работы.

\section{СПИСОК ЛИТЕРАТУРЫ}

1. Adler R. J. An Introduction to Continuity, Extrema, and Related Topics for General Gaussian Processes. Hayward: IMS, 1990, 160 p. (IMS Lecture Notes Monogr. Ser., v. 12.)

2. Anderson T. W., Darling D. A. Asymptotic theory of certain «goodness of fit» criteria based on stochastic processes. - Ann. Math. Statist., 1952, v. 23, p. 193-212.

3. Beghin L., Nikitin Ya., Orsingher E. Exact small ball constants for some Gaussian processes under $L^{2}$-norm. - Зап. научн. сем. ПОМИ, 2003, т. 298, с. 5-21.

4. Bellman R. Adaptive Control Processes: a Guided Tour. Princeton: Princeton Univ. Press, 1961, 255 p.

5. Blum J.R., Kiefer J., Rosenblatt M. Distribution free tests of independence based on the sample distribution function. - Ann. Math. Statist., 1961, v. 32, № 2, p. 485-498.

6. Buslaev A.P., Seleznjev O.V. On certain extremal problems in the theory of approximation of random processes. - East J. Approx., 1999, v. 5, № 4, p. 467-481.

7. Csörgö M., Horváth L. Limit Theorems in Change-point Analysis. Chichester: Wiley, 1997, $414 \mathrm{p}$.

8. Deheuvels $P$. An asymptotic decomposition for multivariate distribution-free tests of independence. - J. Multivariate Anal., 1981, v. 11, p. 102-113.

9. Dugue D. Sur des tests d'indépendence «indépendants de la loi». - C. R. Acad. Sci., 1975 , v. 281 , p. $1103-1104$.

10. Гнеденко Б. В., Колмогоров А.Н. Предельные распределения для сумм независимых случайных величин. М.-Л.: ГТТИ, 1949, 264 с.

11. Henze N., Nikitin Ya. Yu. A new approach to goodness-of-fit testing based on the integrated empirical process. - J. Nonparametr. Statist., 2000, v. 12, p. 391-416.

12. Hoeffding W. A non-parametric test of independence. - Ann. Math. Statist., 1948, v. 19 , p. $546-557$.

13. Koning A. J., Protasov V. Tail behaviour of Gaussian processes with applications to the Brownian pillow. - J. Multivariate Anal., 2003, v. 87, № 2, p. 370-397. 
14. Kühn T., Linde W. Optimal series representation of fractional Brownian sheets. Bernoulli, 2002, v. 8, № 5, p. 669-696.

15. Lifshits M. A., Tulyakova E. V. Curse of dimensionality in approximation of random fields. - Probab. Math. Statist., 2006, v. 26, № 1, p. 97-112.

16. Петров В. В. Суммы независимых случайных величин. М.: Наука, 1972, 416 с.

17. Петров В.В. Предельные теоремы для сумм независимых случайных величин. М.: Наука, 1987, 320 c.

18. Pycke J.-R. Multivariate extensions of the Anderson-Darling process. - Statist. Probab. Lett., 2003, v. 63, № 4, p. 387-399.

19. Karhunen K. Zur Spektraltheorie stochastischer Prozesse. - Ann. Acad. Sci. Fennicae, 1946, № 34, p. 1-7.

20. Karhunen K. Über lineare Methoden in der Wahrscheinlichkeitsrechnung. - Ann. Acad. Sci. Fennicae, 1947, № 37 p. 3-79.

21. Kosambi D. D. Statistics in function space. - J. Indian Math. Soc. (N.S.), 1943, v. 7, p. $76-88$.

22. Лифшии М. А. Гауссовские случайные функции. Киев: ТВіMC, 1995, 246 с.

23. Loève M. Fonctions aléatoires de second ordre. - Revue Sci., 1946, v. 84, p. 195-206.

24. Обухов А. М. Статистическое описание непрерывных полей. - Труды Геофиз. ин-та АН СССР, 1954, № 24 (151), р. 3-42.

25. Пугачев B. С. Общая теория корреляции случайных функций. - Изв. АН СССР, сер. матем., 1953, т. 17, № 5, с. 401-420.

26. Ritter K. Average-case analysis of numerical problems. - Lecture Notes in Math., 2000, v. 1733 , p. $1-254$.

27. Sabelfeld K. Expansion of random boundary excitations for elliptic PDEs. - Monte Carlo Methods Appl., 2007, v. 13, № 5-6, p. 405-453.

28. van der Vaart A. W., Wellner J. A. Weak Convergence and Empirical Processes with Applications to Statistics. New York: Springer-Verlag, 1996, 508 p. (Springer Series in Statistics.)

29. Watson G. S. Goodness-of-fit tests on a circle. - Biometrika, 1961, v. 48, p. 109-114.

30. Woźniakowski $H$. Average case complexity of linear multivariate problems. Part I: Theory. Part II: Applications. - J. Complexity, 1992, v. 8, № 4, p. 337-372; p. 373392.

31. Woźniakowski $H$. Tractability and strong tractability of linear multivariate problems. - J. Complexity, 1994, v. 10, № 1, p. 96-128.

32. Woźniakowski H. Tractability and strong tractability of multivariate tensor product problems. - J. Computing and Information, 1994, v. 4, p. 1-19.

33. Woźniakowski $H$. Tractability of multivariate problems for weighted spaces of functions. - Approximation and Probability. Warsaw: Polish Acad. Sci., 2006, p. 407-427. (Banach Center Publ., v. 72.) 